\title{
Direct probing of evanescent field for characterization of porous terahertz fibers
}

Atakaramians, Shaghik; Afshar V., Shahraam; Nagel, Michael; Rasmussen, Henrik K.; Bang, Ole; Monro, Tanya M.; Abbott, Derek

Published in:

Applied Physics Letters

Link to article, DOI:

$10.1063 / 1.3568892$

Publication date:

2011

Document Version

Publisher's PDF, also known as Version of record

Link back to DTU Orbit

Citation (APA):

Atakaramians, S., Afshar V., S., Nagel, M., Rasmussen, H. K., Bang, O., Monro, T. M., \& Abbott, D. (2011). Direct probing of evanescent field for characterization of porous terahertz fibers. Applied Physics Letters, 98(12), 121104. https://doi.org/10.1063/1.3568892

\section{General rights}

Copyright and moral rights for the publications made accessible in the public portal are retained by the authors and/or other copyright owners and it is a condition of accessing publications that users recognise and abide by the legal requirements associated with these rights.

- Users may download and print one copy of any publication from the public portal for the purpose of private study or research.

- You may not further distribute the material or use it for any profit-making activity or commercial gain

- You may freely distribute the URL identifying the publication in the public portal 


\title{
Direct probing of evanescent field for characterization of porous terahertz fibers
}

\author{
Shaghik Atakaramians, ${ }^{1,2, a)}$ Shahraam Afshar V., ${ }^{2}$ Michael Nagel, ${ }^{3}$ Henrik K. Rasmussen, ${ }^{4}$ \\ Ole Bang, ${ }^{5}$ Tanya M. Monro, ${ }^{2}$ and Derek Abbott ${ }^{1}$ \\ ${ }^{1}$ Adelaide T-ray Group, School of Electrical and Electronic Engineering, The University of Adelaide, \\ SA 5005, Australia \\ ${ }^{2}$ Institute for Photonics and Advanced Sensing, School of Chemistry and Physics, \\ The University of Adelaide, SA 5005, Australia \\ ${ }^{3}$ Institut für Halbleitertechnik, RWTH Aachen University, Sommerfeldstr. 24, 52074 Aachen, Germany \\ ${ }^{4}$ Department of Mechanical Engineering, Technical University of Denmark, \\ DK-2800 Kongens Lyngby, Denmark \\ ${ }^{5}$ Department of Photonics Engineering (DTU Fotonik), Technical University of Denmark, \\ DK-2800 Kongens Lyngby, Denmark
}

(Received 10 January 2011; accepted 25 February 2011; published online 22 March 2011)

\begin{abstract}
We develop a technique based on a micromachined photoconductive probe-tip to characterize a terahertz (THz) porous fiber. Losses less than $0.08 \mathrm{~cm}^{-1}$ are measured in the frequency range from 0.2 to $0.35 \mathrm{THz}$, with the minimum of $0.003 \mathrm{~cm}^{-1}$ at $0.24 \mathrm{THz}$. Normalized group velocity greater than 0.8 , which corresponds to dispersion values in between -1.3 and $-0.5 \mathrm{ps} / \mathrm{m} / \mu \mathrm{m}$ for $0.2<f<0.35 \mathrm{THz}$ are obtained. Moreover, we directly measure the evanescent electric field as a function of frequency. Good agreement between the measured curves and expected theoretical values indicates the low invasiveness of the applied probe-tip. (C) 2011 American Institute of Physics. [doi:10.1063/1.3568892]
\end{abstract}

Terahertz $(\mathrm{THz})$ pulses propagate in free-space with almost zero loss and dispersion, however, waveguides are required when the application point is inaccessible, a stronger interaction of $\mathrm{THz}$ radiation with the sample is required, and it is required to confine the pulse in a subwavelength regime or focus the beam to a smaller spot size to beat the diffraction limit. ${ }^{1-3}$ Porous polymer fibers-air-clad fibers with subwavelength features embedded within the core-have been identified as a means of achieving low loss, low dispersion and high birefringence for guiding the THz pulses. ${ }^{4-6}$ Maintaining the polarization of the propagating field in $\mathrm{THz}$ waveguides - by using asymmetrical subwavelength air holes $^{6,7}$ - makes these fibers a good substitute for free-space, where the polarization state of the $\mathrm{THz}$ field is always preserved. Two types of porous fibers, i.e., with symmetrical and asymmetrical features, have been fabricated exploiting the extrusion technique. ${ }^{7}$

The low-dispersion characteristics of the polymer porous fibers compared with their microwire counterparts and the birefringence characteristics $(\cong 0.012$ at $0.65 \mathrm{THz}$ ) have been confirmed experimentally by Atakaramians et al., ${ }^{7}$ using the cut-back method. In this approach three different lengths of a porous fiber were employed for characterization, and the fibers were directly positioned on the emitter and detector. It was identified that the quality and repeatability of the cleaved end-face of the porous fibers, their slight bending and alignment introduce high uncertainty in the loss measurements. In order to conduct a sufficiently precise cutback-based loss measurement a rapid, reproducible, and in situ cleaving method is required. This can be achieved utilizing an ultraviolet laser, ${ }^{8}$ which leads to complicated experimental setup and enormous effort. A directional coupler based method, where a secondary waveguide is located to

${ }^{\text {a)}}$ Electronic mail: shaghik@eleceng.adelaide.edu.au. and translated along the fiber being characterized, is an approach that eliminates cleaving of the fiber. ${ }^{9}$ However, there are disadvantages in that the coupling efficiency and length of the coupler is frequency dependent, which complicates the interpretation of the results. Furthermore, a bolometer is employed as a detector, hence, fiber dispersion properties are still not accessible. Average losses as low as $0.01 \mathrm{~cm}^{-1}$ have been reported. ${ }^{9}$

Here, we demonstrate a technique in which we use a micromachined photoconductive probe-tip to directly sample the evanescent $\mathrm{THz}$ electric field in the time-domain at the vicinity of the fiber surface and hence measure simultaneously both absorption coefficient $\left(\alpha_{\text {eff }}\right)$ and effective refractive index $\left(n_{\text {eff }}\right)$, from which group velocity and dispersion can be calculated, of the propagating modes of a porous fiber. This approach enables us to accurately explore the radial distribution of evanescent field in the air-clad of these porous fibers as a function of frequency. We present the experimental results and demonstrate a good agreement with the numerical results, based on structural information obtained from scanning electron microscope (SEM) image of the fabricated porous fiber.

The schematic of the experimental setup used for characterization of porous fibers is shown in Fig. 1. A modelocked Ti:sapphire laser with a pulse width of less than $100 \mathrm{fs}$, central frequency of $800 \mathrm{~nm}$ and a repetition rate of $80 \mathrm{MHz}$ is used to drive the emitter and detector. A photoconductive antenna array ${ }^{10}$ consisting of ten antenna elements on a $1.7 \mathrm{~mm}$ thick silica substrate is used for generation of THz pulses, while a photoconductive switch with a pair of tapered electrodes on a triangular-shaped low temperature grown gallium arsenide substrate ${ }^{11}$ (probe-tip) is utilized for sampling of $\mathrm{THz}$ pulses. The probe-tip, shown in Fig. 1, allows sampling of the $\mathrm{THz}$ pulses along the waveguide. This is possible because the evanescent field of the 


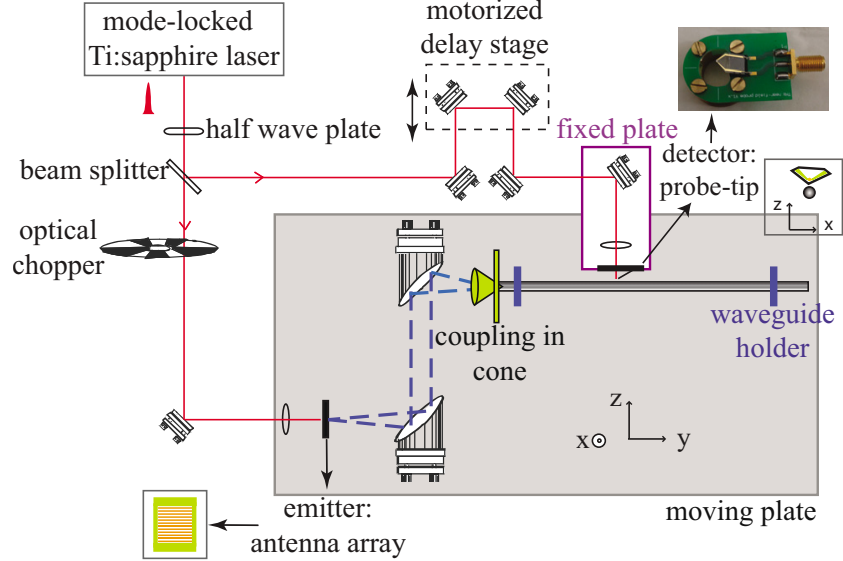

FIG. 1. (Color online) Schematic of the $\mathrm{THz}$ time-domain spectroscopy setup employed for characterization of porous fibers. Two parabolic mirrors and a metallic cone are employed to couple the $\mathrm{THz}$ pulses into the waveguide. The emitter and detector are shown in the inset.

mode propagating along the porous fiber expands into the air-cladding region beyond the fiber itself. Therefore, instead of using three different fibers, or the cut-back method, the propagating mode is directly sampled on three different positions along the waveguide.

To increase the coupling efficiency of $\mathrm{THz}$ pulses into the fiber and to block the stray beams, a metallic cone with an $800 \mu \mathrm{m}$ pin-hole at the vertex is employed. The waveguide is mounted on two specially designed plastic holders, which have low interference with the propagating mode. The plastic holder consists of a $1.5 \mathrm{~cm}$ plastic ring with three slim struts joining at the center of the ring. The waveguide is glued to the center of the holders at both ends and is positioned on a three-dimensional manual moving stage. This approach keeps the fiber straight and minimizes the losses due to the curvature of the fiber (bend loss). Keeping the distance between the probe-tip and waveguide constant is the major challenge of this approach. Thus we used two cameras to monitor the alignment of the probe-tip and waveguide. The probe-tip distance from the waveguide is monitored by a camera from the bird's eye view. For each position the probe-tip is aligned so that it gently touches the waveguide. The alignment of the waveguide with the probe-tip in the $x$ direction is also monitored by another camera located in the same plane with the waveguide and probe-tip. This second camera is used to make sure that the waveguide is aligned at the center of probe-tip. For sampling of $\mathrm{THz}$ pulses with the probe-tip arrangement shown in Fig. 1, it is required that the laser beam to be polarized in the $x$ direction. Therefore, a half wave plate is used to rotate the polarization of the linearly polarized laser beam ( $z$ direction) in the $x$ direction. In this experiment, we use a $600 \mu \mathrm{m}$ diameter porous fiber, namely, spider-web porous fiber, as shown in the insets of Figs. 2(a) and 3. The fiber is made of cyclic-olefin copolymer (COC). The emitter, focusing optical lens, and waveguide are situated on a sliding table and the detector is kept still, Fig. 1. The moving plate is translated to the left and three positions with $50 \mathrm{~mm}$ separations are sampled.

Figures 2(a) and 2(b) show the measured $\alpha_{\text {eff }}$ and $n_{\text {eff }}$ curves. The noise floor of the system is defined by measuring the spectral amplitude when the waveguide is removed from the system. The $\alpha_{\text {eff }}$ and $n_{\text {eff }}$ of the propagating mode is the average of $\alpha_{\text {eff }}$ and $n_{\text {eff }}$ values obtained by the comparison of
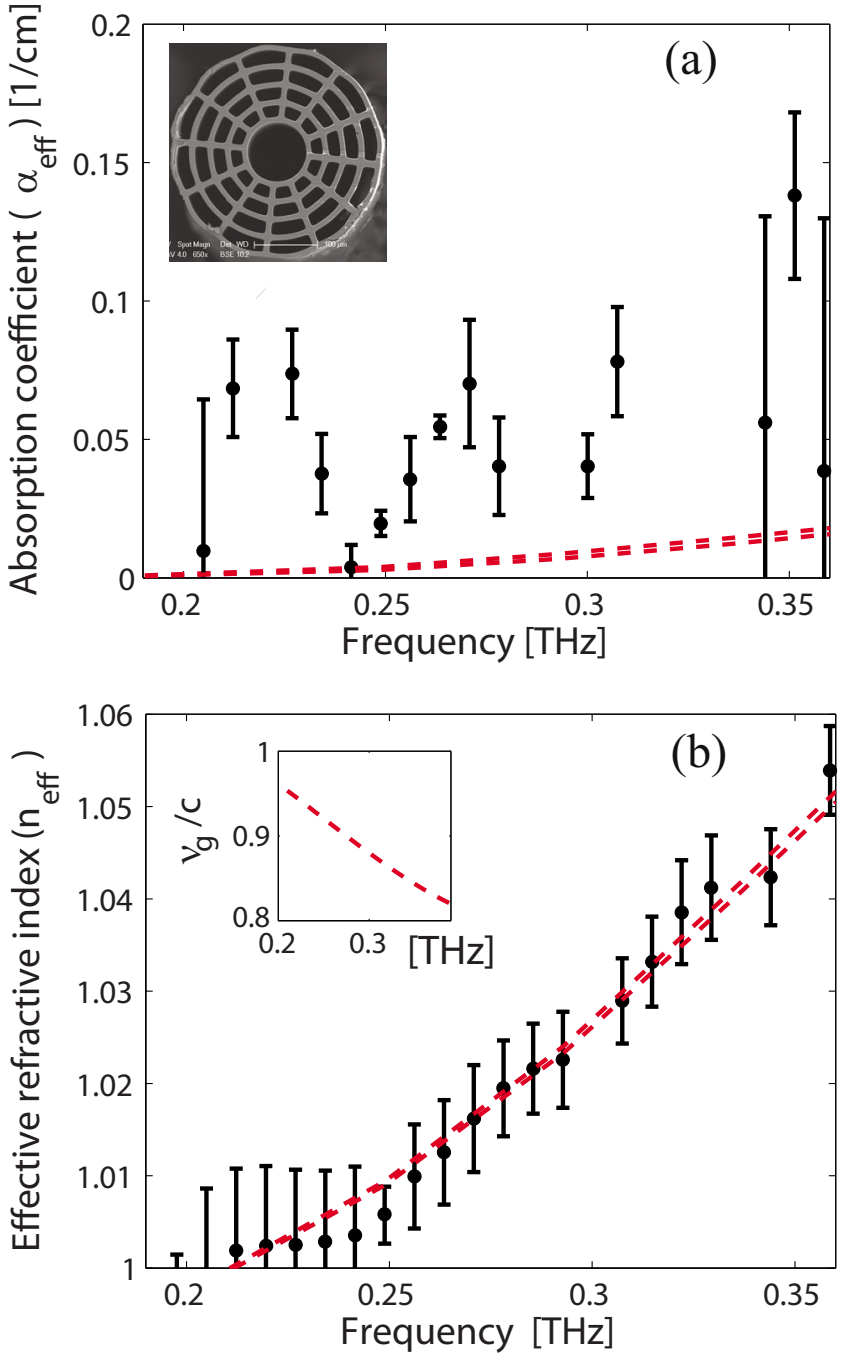

FIG. 2. (Color online) (a) Absorption coefficient, $\alpha_{\text {eff }}$, and (b) effective refractive index of the propagating fundamental mode in a $600 \mu \mathrm{m} \mathrm{COC}$ spider-web porous fiber: black dots and red dashed lines represent the measured and theoretical values, respectively. The fiber cross-section and group velocity are shown in the inset of parts (a) and (b), respectively.

the pulses sampled at three positions. Theoretical $\alpha_{\text {eff }}$ and $n_{\text {eff }}$ curves of the real porous fiber-calculated based on the SEM image of the fiber and using a commercial finite element package COMSOL 3.5-are also depicted with red dashed lines. The slight deformation of the fiber crosssection during the fiber drawing introduces birefringence less than 0.001 . The theoretical results of the both polarizations are illustrated in Figs. 2(a) and 2(b). The error bars in Fig. 2 represent uncertainty of the $\alpha_{\text {eff }}$ and $n_{\text {eff }}$ measurements due to two major sources: Length and signal variation during the scans. For the length uncertainty, we consider $\pm 1 \mathrm{~mm}$ error in the values recorded from a ruler. These sources of error are independent, thus the total uncertainty for the measurements is equal to the quadrature sum of uncertainties obtained from two sources described above. Absorption coefficients less than $0.08 \mathrm{~cm}^{-1}$ are measured at $0.2-0.35 \mathrm{THz}$, with the minimum of $0.003 \mathrm{~cm}^{-1}$ at $0.24 \mathrm{THz}$. The measured effective refractive index curve follows closely the expected theoretical values, i.e., $n_{\mathrm{eff}}<1.05$ for $0.2<f<0.35 \mathrm{THz}$. This leads to normalized group velocities $\left(\nu_{\mathrm{g}} / c\right)$ greater than 0.8 , which correspond to dispersion values in between -1.3 and $-0.5 \mathrm{ps} / \mathrm{m} / \mu \mathrm{m}$ for $0.2<f<0.35 \mathrm{THz}$, with zero- 


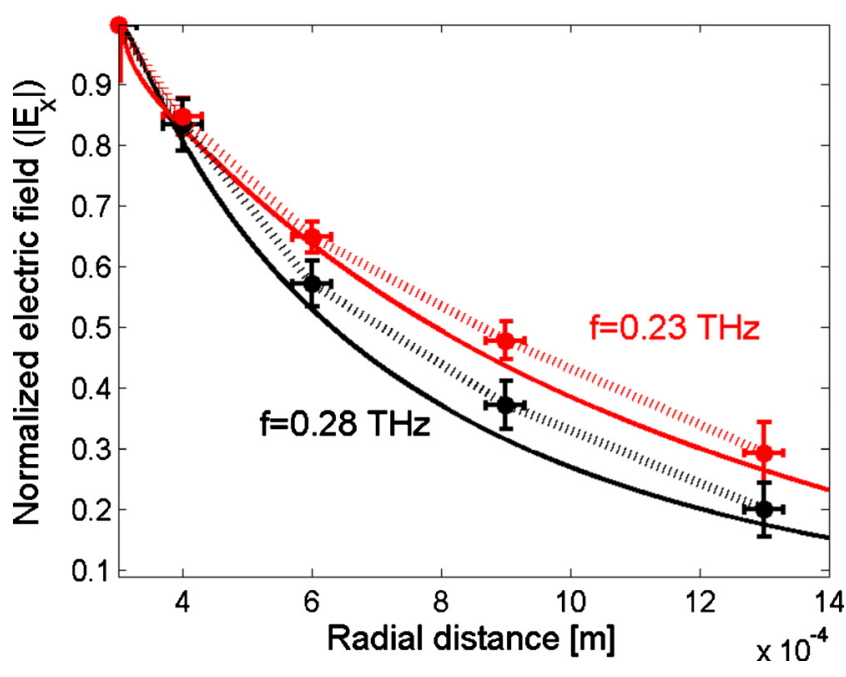

FIG. 3. (Color online) Measured radial field distribution from the waveguide interface at $0.23 \mathrm{THz}$ (top dotted line), and $0.28 \mathrm{THz}$ (bottom dotted line). The solid lines represent the theoretical values (for a $600 \mu \mathrm{m}$ diameter real porous fiber) of normalized radial distribution of $x$-component of the electric fields.

dispersion estimated at $0.5 \mathrm{THz}$. The negative dispersion values indicate normal dispersion.

In order to further demonstrate the sampling capability of the probe, we measure the frequency-dependent radial field distribution of a COC spider-web porous fiber. For this purpose, the probe-tip has been translated away (along the radial direction) from the fiber and the $\mathrm{THz}$ pulses are measured at different radial positions. Figure 3 shows the measured radial electric field distribution at 0.23 and $0.28 \mathrm{THz}$. The fields are normalized to the respective maximum amplitude at the porous fiber surface. The curves indicate that increasing the frequency increases the confinement of the field to the fiber, as expected. For comparison, the theoretical values of the normalized radial distribution of the electric fields of a real porous fiber are calculated and shown with solid lines. According to the measurements, the diameter of the waveguide under investigation varies from 570 to $640 \mu \mathrm{m}$. Thus, a $600 \mu \mathrm{m}$ diameter porous fiber is considered for the theoretical calculation.

The vertical error bars in Fig. 3 represent the standard variations in the signal alteration during the measurement. The horizontal error bars represent the total standard deviation due to the elliptical shape of the waveguide, and polarization state at the measurement point. The real waveguide has a slightly elliptical cross-section as shown in the inset of Fig. 3. The measurement can be conducted starting from any location of this elliptical surface. Moreover, the elliptical surface results in birefringence, i.e., the effective refractive index of the mode continuously varies with the electric field orientation angle between maximum and minimum values that are spaced by $90^{\circ}$. The polarization state of linearly polarized $\mathrm{THz}$ pulse changes from linear to elliptical and circular as the pulse propagates along the waveguide. Thus, the detector at any location along the waveguide samples the projection of the electric field along the probe-tip, which can be $x$ - or $y$-components or the total electric field of the propagating mode, depending on the field polarization at the probe-tip location. There is a subtle difference in the decay rate of these three normalized electric fields. The $x$-component of the electric field is depicted and the difference is included as an error source in the measured curves on the horizontal axis. The good agreement between the measured curves and expected theoretical values indicates the low invasiveness of the applied probe-tip. In order to observe the effect of the presence of the probe-tip, a further investigation is required.

In conclusion, we have developed a technique by exploiting a micromachined photoconductive probe-tip for characterization of $\mathrm{THz}$ porous fibers. Losses less than $0.08 \mathrm{~cm}^{-1}$ are measured at the frequency range from 0.2 to $0.35 \mathrm{THz}$, with the minimum of $0.003 \mathrm{~cm}^{-1}$ at $0.24 \mathrm{THz}$. Normalized group velocity greater than 0.8 , which is equivalent to dispersion values in between -1.3 and $-0.5 \mathrm{ps} / \mathrm{m} / \mu \mathrm{m}$ for $0.2<f<0.35 \mathrm{THz}$ is obtained. Moreover, we measure the evanescent electric field distribution of the porous fiber as a function of frequency, which follows the trend of expected theoretical values. This technique is applicable for the characterization of any waveguide that contains an extended propagating mode that is accessible to measurement along the waveguide.

This research was supported under the Australian Research Council's Discovery Projects Funding Scheme (Grant Nos. DP0556112 and DP0880436). We gratefully acknowledge Dr Heike Ebendorff-Heidepriem and Dr Bernd M. Fischer for useful discussions. Roger Moore for fiber drawing and Henry Ho for T-ray laboratory hardware assistance. T. M. Monro acknowledges the support of an ARC Federation Fellowship.

${ }^{1}$ J. S. Melinger, N. Laman, S. Sree Harsha, and D. Grischkowsky, Appl. Phys. Lett. 89, 251110 (2006).

${ }^{2}$ S. A. Maier, S. R. Andrews, L. Martin-Moreno, and F. J. Garcia-Vidal, Phys. Rev. Lett. 97, 176805 (2006).

${ }^{3}$ M. Nagel, M. Först, and H. Kurz, J. Phys.: Condens. Matter 18, S601 (2006).

${ }^{4}$ A. Hassani, A. Dupuis, and M. Skorobogatiy, Opt. Express 16, 6340 (2008).

${ }^{5}$ S. Atakaramians, S. Afshar V., B. M. Fischer, D. Abbott, and T. M. Monro, Opt. Express 16, 8845 (2008).

${ }^{6}$ S. Atakaramians, S. Afshar V., B. M. Fischer, D. Abbott, and T. M. Monro, Opt. Commun. 282, 36 (2009).

${ }^{7}$ S. Atakaramians, S. Afshar V., B. M. Fischer, M. Nagel, H. EbendorffHeidepriem, D. Abbott, and T. M. Monro, Opt. Express 17, 14053 (2009).

${ }^{8}$ S. Atakaramians, K. Cook, H. Ebendorff-Heidepriem, S. Afshar V., J. Canning, D. Abbott, and T. M. Monro, IEEE Photonics Journal 1, 286 (2009).

${ }^{9}$ A. Dupuis, J.-F. Allard, D. Morris, K. Stoeffler, C. Dubois, and M. Skorobogatiy, Opt. Express 17, 8012 (2009).

${ }^{10}$ D. Saeedkia, R. R. Mansour, and S. Safavi-Naeini, IEEE Trans. Antennas Propag. 53, 4044 (2005).

${ }^{11}$ M. Wächter, M. Nagel, and H. Kurz, Appl. Phys. Lett. 95, 041112 (2009) 